\title{
Introduction of Questionnaires for Quality of Life of Patients with Malignant Tumors of the Central Nervous System into Neurosurgical Practice in the Republic of Kazakhstan
}

\author{
Serik Akshulakov ${ }^{1}$, Nurgul Aldiyarova ${ }^{1}$, Nurzhan Ryskeldiyev ${ }^{1}$, Zauresh \\ Akhmetzhanova $^{1,2 *}$, Kamila Gaitova ${ }^{2}$, Raushan Auezova ${ }^{1}$, Aidos Doskaliyev ${ }^{1}$, \\ Talgat Kerimbayev ${ }^{1}$
}

\begin{abstract}
Background: Studies of quality of life (QoL) of oncological patients is carried out using questionnaires approved in many international clinical studies. The European Organization for Research and Treatment of Cancer EORTC QLQ-C30 (Quality of Life Questionnary-Core 30) and its special brain cancer module EORTC QLQ-BN20 are widely used in the world neurooncologic practice. They are available in more than 80 official versions of 30 languages of the world. Previously we used the official versions in Russian, which often causes difficulty in understanding for native Kazakh language speakers, who comprise more than $60 \%$ of our respondents. This was the reason for creating a version of Kazakh language. Therefore, in 2014 for the first time the process of adaptation of questionnaires to the Kazakh language was initiated. Materials and Methods: The translation process of questionnaires to Kazakh language was held in accordance with the requirements of the European Organization for Research and Treatment of Cancer EORTC on QoL and consisted of the following stages: preparation - translation - pilot testing - approval. The official permission of authors and "Guideline on translation" was obtained which was developed by the working group of the EORTC on QoL. The pilot testing of EORTC QLQ-C30 and QLQ-BN20 questionnaires was conducted on the basis of the Department of Central Nervous System Pathology of the "National Centre for Neurosurgery" in patients with malignant tumors of the central nervous system. Results: The official versions of the EORTC QLQ-C30 and QLQ-BN20 questionnaires in Kazakh language were introduced and adapted in practical neurosurgical operations in Kazakhstan. Conclusions: The approved versions of the questionnaires in Kazakh language are now available for mainstream use on the official website EORTC.com. The versions of these questionnaires can be used in domestic cohort studies and clinical practice in the Republic of Kazakhstan. The use of these tools for assessing QoL will help professionals in the planning of individual treatment strategies and selection of the necessary therapy.
\end{abstract}

Keywords: CNS malignant tumors - quality of life - EORTC questionnaires - Kazakhstan language

Asian Pac J Cancer Prev, 17 (2), 873-876

\section{Introduction}

Annually in Kazakhstan more than 30,000 patients revealed with various malignancies and about 150,000 patients under the supervision of oncological institutions (Tuleutayev, 2012). Only in Astana every year more than 1,200 people become ill and more than 500 die from various malignancies. Among newly diagnosed cases only $49.5 \%$ identified as the stage $1-2$, and $50 \%$ of cases are extended forms of the disease. The average annual incidence rate of malignant tumors of the central nervous system (MT CNS) in Kazakhstan for the period 2004-2011 was 3.7 per 100,000 populations (Igissinov et al., 2013). In dynamics, the annual incidence increased from period of 2004 from 3.34 per 100,000 populations to 3.90 in 2011. The average age of patients was 44.5 years, i.e. a very high percentage of disease among people of working age. The incidence of primary malignant and benign brain tumors in the United States for the period 20052009 , amounted to 20.6 cases per 100,000 populations according to the date of the Central Brain Tumor Registry of the United States (CBTRUS, 2005-2009). Among them 7.3 cases occur in malignant tumors and benign in 13.3 cases. Incidence in women is 22.3 and for men 18.8 per 100,000 populations. According to the international data, the rates of 5-year survival in malignant brain tumors vary considerably depending on the histological subtype and $79.1 \%$ for oligodendrogliomas, $27.4 \%$ for anaplastic 
astrocytomas and $4.5 \%$ for glioblastomas (Ohgaki et al., 2005; Akshulakov et al., 2014).

Glial brain tumor is the most common in the group of malignant brain tumors, accounting for up to $50 \%$ of all tumors of the CNS and have very poor prognosis. Compared with lung cancer or breast cancer it is relatively uncommon malignant tumor, but nevertheless, due to its aggressive nature and direct CNS affect it has disproportionately greater percentage of morbidity and mortality with serious influence on the healthcare system in general, and especially on the patients themselves, their families and loved ones (Tuleutayev, 2012). Also one of the important factors is that the clinical manifestations of this tumor are varied and can appear actually suddenly in the setting of the overall health, and they are accompanied by severe neurological deficits caused by compression of the brain tissue, often with seizures, focal symptoms, can cause a stroke, causing deep anxious-depressive disorders, which significantly reduce quality of life of patients (Ohgaki et al., 2005; Akshulakov et al., 2014). The initial stages of the disease may also present psychiatric symptoms which assigned to 7 main categories, such as depressive symptoms, apathy, manic symptoms, psychosis, personality changes, eating disorders, and a miscellaneous category for the less frequently encountered symptoms (Madhusoodanan et al., 2015). These neurooncological patients tend to have low levels of self-service, need the additional care and attention both of family and of the medical and social services. For them, the goal of treatment is not only an extension of life, but also maintaining a satisfactory quality of life as long as possible (Ohgaki et al., 2005). In recent years, Kazakhstan has done a great job to improve the provision of specialized medical care - neurosurgical, oncological, neurological care - to neurooncological patients, but the issue of psychological support for patients and their relatives and friends is still open. Everyone knows that an important condition for overcoming this terrible disease is the psychological state of the patient and his/her inner spirit of recovery, as well as the support of relatives, who themselves often remain in the shock state from the heard diagnosis (Lee et al., 2015). It is not an easy problem, and correspondingly a study of quality of life in neurooncological practice is an urgent task.

Each of us has ever faced with the concept of quality of life, but for everyone this concept means different things. For some it is an opportunity to travel to different countries, for others it is an opportunity to do what he/she likes, but for some it's an elementary self-service. Quality of life is individual and depends on the lifestyle of the person, the experience of previous years, hopes for the future, dreams and ambitions (Goerling and Stickel, 2014). Aristotle on the fundamental problems of the quality of life said that "often the same person changes his mind: when he becomes ill, it is health, and as long as he is healthy it is money". The term "quality of life" in medicine came from sociology, which is understood as the individual's ability to function in society according to his/her status and get the satisfaction from it (Yeliseyeva, 2003). In 1993, the World Health Organization gave the following definition: Quality of life (QoL) is the perception of individuals of

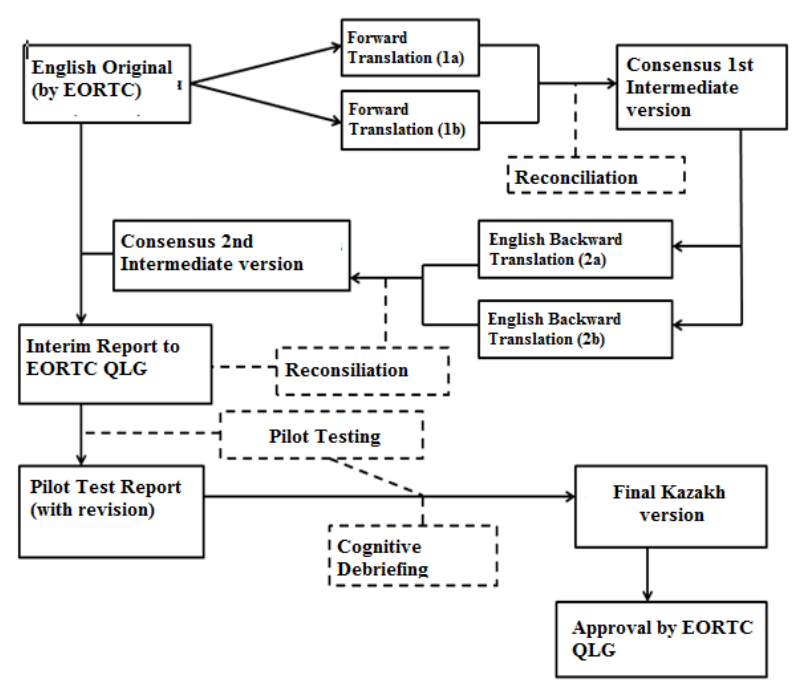

Figure 1. Translation Process of the EORTC QLQ into the Kazakh Language

their status in life in the context of the culture and values of the environment in which they live, according to their goals, expectations, standards and concerns (WHO, 1993). Quality of life includes information on the major area of life: psychological, physical, social, economic, spiritual, which allows determining the differential impact of the disease and treatment on the patient's condition (Bottomley et al., 2005).

The earliest surveys of quality of life were focused on an assessment of the physical condition of the patient. So, the famous American oncologist David A. Karnofsky developed the index in 1948, allowing doctor to evaluate the patient's physical condition from a scale of $100 \%$ at full (normal) activity to $0 \%$ (fatality of general condition), which is still used today and is named "Karnofsky Performance Status Scale" in his honor (Karnofsky and Burchenal, 1949). In 1981, Spitzer proposed a different scale to assess the quality of life, where the doctor acts as an observer and evaluates not only the physical condition of the patient, but also his/her psychological, social and spiritual aspects of life (Spitzer, 1981). Today, self-priming questionnaires by patient are more suitable and reliable for the assessment of QoL. According to the modern requirements, they should be short and at the same time should have psychometric properties such as reliability, validity and sensitivity. Reliability is a measure of the accuracy and consistency of measurements. Validity is an opportunity to reflect the characteristic which is intended to measure with the help of a questionnaire. Sensitivity is an opportunity to detect changes or differences in quality of life in accordance with changes in the patient's condition or with differences in status of groups of patients with the help of a questionnaire (Bottomley et al., 2005; Budrukkar et al., 2009).

In an effort to improve the study of the quality of life problems, many well-known institutions have created a group on research and compilation of various questionnaires. The most popular of these questionnaires are the American questionnaire Functional Assessment of Cancer Therapy: General (FACT-G); the questionnaire of the European Organization for Research and Treatment of 
Cancer (EORTC QLQ-C30); Rotterdam Scale of Quality of Life (RSQL); CAncer Rehabilitation Evaluation System--short form (CARES - SF). At the conference of the US National Cancer Institute (NCI) and the American Society of Clinical Oncology (ASCO) in 1990 it was stated that the quality of life is the second most important criterion for assessing the results of cancer treatment after survival and is more important than the primary "tumor response" to therapy (Aaronson et al., 1991; Bottomley et al., 2005).

Questionnaire EORTC QLQ-C30 (Quality of Life Questionnary-Core 30) of the European Organization for Research and Treatment of Cancer is the most appropriate to assess the quality of life in our conditions, as it is approved in many international clinical studies (Aaronson et al., 1993; Aaronson et al., 1994; Taphoorn et al., 2013) which have shown its applicability to a variety of cultures. It meets the criteria developed for the questionnaires used in oncology - the criteria of reliability, validity and feasibility. This questionnaire is highly sensitive and useful for assessing the quality of life of patients regardless of the type of cancer (Liu et al., 2009; http://groups.eortc. be). The questionnaire EORTC QLQ-C30 includes 30 questions and consists of:

- 5 functional scales: physical, role, cognitive, emotional and social;

- 3 scales of symptoms: fatigue, nausea \& vomiting, and pain;

- global health status/QoL scale.

There is also a special add-on module, designed and approved for patients with a tumor of the brain EORTC QLQ-BN20 (Therasse, 1996; Maringwa et al., 2011), which includes 20 questions to identify visual disorders, movement disorders, various symptoms of disease, treatment toxicity and uncertainty about the future. Both the questionnaires EORTC QLQ-C30 and QLQ-BN20 are widely used in world neurooncologic practice and presented in more than 80 versions of the 30 official languages of the world, including in Russian.

In the process of assessment of QoL of patients, we realized the need to create a version in Kazakh language, and in 2014 for the first time we initiated the process of adaptation of questionnaires in Kazakh language.

The translation process of questionnaires carried out in accordance with the requirements of the European Organization for Research and Treatment of Cancer on QoL and consisted of the following stages: preparation translation - pilot testing - approval.

During the preparation stage the official permission of authors and Translation guidelines were obtained which was developed by the working group of the EORTC on QoL.

At the stage of the translation of questionnaires, the process was as follows: first of all, primary translation of the original English version of the questionnaire to the Kazakh language was carried out by two translators, who were native Kazakh speakers fluent in English, independently of each other. Then both translations were compared and discussed among translators in attendance of doctors. On the basis of the discussion a single version was accepted, which was later translated back into English by two other translators, also fluent in both languages, working independently of each other. All these steps were documented and sent to the project coordinator of the EORTC, which in turn analyzed and sent questions and comments that arose during the audit. All comments were considered with making appropriate adjustments, then a preliminary version of the questionnaire in Kazakh language was formulated, and the permission to conduct a pilot testing was obtained.

Pilot testing was conducted in two stages among patients with a diagnosis of malignant tumors of the CNS (MT) who are ethnic Kazakh speakers. The success rate of the passage of the pilot test was the lack of controversial, embarrassing or difficult issues for patients. The study was conducted on the basis of the Department of central nervous system pathology of the "National Centre for Neurosurgery" in Astana city and Neurosurgery department of Municipal Clinical Hospital №7 in Almaty city.

Criteria for inclusion of patients in the pilot testing and research of psychometric properties by the version of the EORTC QLQ-BN20 questionnaire in Kazakh language were:

- diagnosis of MT CNS, preliminary or confirmed histologically;

- age over 18 years;

- Kazakh language;

- the absence of concomitant cancer process;

- the absence of concomitant severe physical disease or mental disorder.

After the first pilot testing the minor adjustments in accordance with the comments of respondents have been made. In consequence of the second pilot testing no remarks have been identified. Report for the working group of the EORTC on QoL was developed at the end of each stage of pilot testing.

Approval of the questionnaire. As a result of this work, the final report was formed for the working group of the EORTC on QoL, after which compliance with all the requirements of the process of translation was confirmed and the final version of the questionnaire was formed. The approved version of the questionnaire is now available for mainstream use on the official website EORTC.com (Therasse, 1996).

Thus, the version of the questionnaire EORTC QLQ-C30 and QLQ-BN20 in Kazakh language can be used in domestic cohort studies and clinical practice. In the future, we plan to more thoroughly investigate the quality of life in patients with MT CNS using these questionnaires at different stages of the disease, depending on the received chemo-radiation treatment.

We hope that the questionnaires embedded in the activities of practitioners will contribute to the study of quality of life of neurooncological patients in order to improve individual treatment strategies, the selection of necessary therapy, and timely correction of irregularities in the psychological sphere. And for health care managers it will be one of the tools giving the possibility to analyze the cost-effectiveness of health care, as well as issues relating to disability examination. And most importantly it 


\section{Serik Akshulakov et al}

will improve the quality of life of patients and implement a well-known principle of "Treat the patient, not the disease!"

\section{References}

Aaronson NK, Meyerowitz BE, Bard M, et al (1991). Quality of life research in oncology. Cancer, 67, 839-43.

Aaronson NK, Ahmedzai S, Bergman B, et al (1993). The European Organisation for Research and Treatment of Cancer QLQ-C30: A quality of life instrument for use in international clinical trilas in oncology. J Natl Cancer Inst, 85, 365-75.

Aaronson NK, Cull A, Kaasa S, et al (1994). The European Organisation (or Research and Treatment of Cancer (EORTC) modular approach to quality of life assessment in oncology. Int J Ment Health, 23, 75-96.

Akshulakov S, Igissinov N, Aldiyarova N, et al (2014). Oneyear survival rate of patients with primary malignant central nervous system tumors after surgery in Kazakhstan. Asian Pac J Cancer Prev, 15, 6973-6.

Bottomley A, Flechtner H, Efficace F, et al (2005). Health related quality of life outcomes in cancer clinical trials. Eur J Cancer, 41, 1697-709.

Budrukkar A, Jalali R, Dutta D, et al (2009). Prospective assessment of quality of life in adult patients with primary brain tumors in routine neurooncology practice. $J$ Neurooncol, 95, 413-9.

Central Brain Tumor Registry of the United States analyses of the NPCR and SEER data (2005-2009). Available from: website: www.cbtrus.org.

Goerling U, Stickel A (2014). Quality of life in oncology. Recent Results Cancer Res, 197, 137-52.

Igissinov N, Akshulakov S, Igissinov S, et al (2013). Malignant tumors of the central nervous system in Kazakhstan incidence trends from 2004-2011. Asian Pac J Cancer Prev, 14, 4181-6.

Karnofsky DA, Burchenal JH (1949). The clinical evaluation of chemotherapeutic agents in cancer. In: MacLeod CM (ed) Evaluation of chemotherapeutic agents. Columbia University Press, New York, 196.

Lee H, Park E, Kim S, et al (2015). Quality of life of family members living with cancer patients. Asian Pac J Cancer Prev, 15, 6913-7.

Liu R, Page M, Solheim K, et al (2009). Quality of life in adults with brain tumors: Current knowledge and future directions. Neuro Oncol, 11, 330-9.

Madhusoodanan S, Ting M, Farah T, et al (2015). Psychiatric aspects of brain tumors: A review. World J Psychiatry, 15, 273-85

Maringwa J, Quinten C, King M, et al (2011). Minimal clinically meaningful differences for the EORTC QLQ-C30 and EORTC QLQ-BN20 scales in brain cancer patients. Ann Oncol, 22, 2107-12.

Ohgaki H, Kleihues P (2005) Population-based studies on incidence, survival rates, and genetic alterations in astrocytic and oligodendroglial gliomas. J Neuropathol Exp Neurol, 64, 479-89.

Spitzer WO, Dobson AJ, Hall J, et al (1981). Measuring the quality of life of cancer patients: a concise QL-index for use by physicians. J Chronic Dis, 34, 585-97.

Taphoorn MJB, Sizoo EM, Bottomley A (2013). Review on quality of life issues in patients with primary brain tumors. J Clinical Oncol, 31, 65-72.

Therasse P (1996). A practical guide to EORTC (European Organization for Research and Treatment of Cancer) studies. EORTC Data Center, Brussels.
Tuleutayev ME (2012). Oncological service of Astana city and development priorities. Med J Astana, 3, 14-16.

Yeliseyeva II (2003). Sociology. ECOS, Moscow, 656.

WHO (1993). Study protocol for the World Health Organisation project to develop a quality of life assessment instrument (WHOQOL). Qual Life Res, 2, 153-9. 